\title{
A COMPARISON OF LUMINOSITY CALIBRATIONS FOR MK CLASSIFICATIONS OF OB STARS
}

\author{
Janet Rountree Lesh \\ Laboratory for Astronomy and Solar Physics, NASA
}

It has been apparent for some time that there is a need for a single luminosity calibration to be used with modern MK types for early-type stars, at least from $O$ through middle $B$. The widely used calibration of Blaauw (1963) has to be replaced because the refinement of the MK system - as reflected in the large collections of spectral types by Lesh (1968), Hiltner, Garrison, and Schild (1969) and Walborn (1971) - has led to a lower mean luminosity for most main sequence subgroups of early-type stars, as the higher luminosity stars tend to move out of class $V$. Thus the calibrations of Lesh (1968) and Walborn (1972, 1973) are systematically fainter than Blaauw's

We recognize, of course, that a luninosity calibration based on real standard stars need not be capable of representation by a smooth curve. However, the spectral classifications should be closely enough tied to physical parameters so that the calibration is at least monotonic. The problems that can arise in the absence of such a self-consistent calibration are illustrated by Snow and Morton (1976), who had difficulty in finding a unique luminosity limit between stars that show mass loss and those that do not.

In our present remarks, we shall be concerned only with stars of luminosity classes III, IV, and $V$ since there are not many new data on supergiants. The principal recent luminosity calibrations for $D$ and B type stars are those of Lesh (1968), Walborn (1972, 1973) and Conti and Alschuler (1971). Conti and Alschuler's calibration goes only from 06.5 to 09.5 for giants and main sequence stars, and in this region it is essentially identical to Walborn's calibration - the maximum difference between them is $0.2 \mathrm{mag}$, with $0.1 \mathrm{mag}$ more typical. 
Ideally, we would like to adopt Walborn's calibration for the 0 stars because his classification introduced luminosity classes for these stars. Lesh's classification (which involved only a few 0 stars) was made at a time when the MK system did not include luminosity classes for 0 stars, so her calibration is actually an average of all luminosity classes for these types. On the other hand, Lesh's calibration for B stars is based on many more standards than Walborn's and hence is to be preferred. The problem is to fit the two calibrations together at a suitable point, in such a way that there is no discontinuity.

For luminosity classes III and IV, there is no difficulty. The two claibrations match up well at BO, and we use Walborn's $\mathrm{M}_{\mathrm{v}}$ for earlier types and Lesh's $M_{\mathrm{V}}$ for later ones. (Actually, Walborn does not give explicit values for class IV earlier than 09 - we interpolate between his values for class III and class V.) The only real problem arises for the class $V$ stars. Here the two calibrations are very different from 09 through $B 1$, as shown in Fig. 1. Making a transition at any of these points would result in a discontinuity, in the sense that a later spectral type would have a higher luminosity than an earlier one. We do not advise simply averaging the two calibrations on this region, for this would have no physical basis - and it would not A COMPARHSON OF CLASS 7 CALERATIONS necessarily solve the problem.

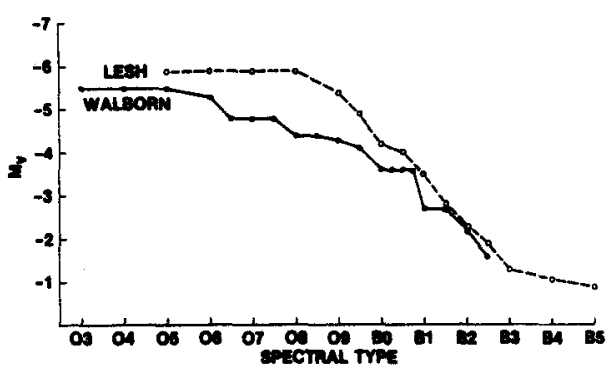

Fig. 1. A comparison of class V calibrations

Instead, we have examined, case by case, the standard stars used in the two calibrations for type 09 to Bl, to find out, if possible, the source of the difference and to determine the best $\mathrm{M}_{\mathrm{v}}$ for each type.

09: Walborn's value is an average of three stars, including 10 Lac. Lesh's value is based only on 10 Lac, whose photometry apparently disagrees with its association distance. We adopt Walborn's Mv.

09.5: Walborn's value is based on +600 449, while Lesh's is based on oOri $A$. The latter may have discrepant photometry because of its multiplicity, so we adopt Walborn's $\mathrm{M}_{\mathrm{y}}$. 
B0-B0.7: Walborn's value for all four of these types is an average of one star of each type: respectively $\cup$ Ori, $\tau$ Sco, HD 36960, and 40 Per. Lesh's value for $B 0$ is composed of $U$ Ori and $\tau$ Sco, and for BO.5 of $\mathrm{HD} 24131$, HD 36960, and B Sco AB. Both $\tau$ Sco and $U$ Ori have photometry that is quite discrepant with their association distances, but the association distance for $u$ Ori is much better determined. We recommend discarding $\tau$ Sco and using the association distance for $U$ Ori to get $M_{v}$ ( $B O \mathrm{~V}$ ); for $B 0.5 \mathrm{~V}$, we use the association distance to get a new $M_{v}$ for $H D 36960$, and average this with $\mathrm{HD} 24131$ and $B$ Sco. We make a linear interpolation for the intermediate types $\mathrm{B} 0.2 \mathrm{~V}$ and $\mathrm{B} 0.7 \mathrm{~V}$.

B1: Walborn's $M_{y}$ is based only on $w^{1}$ Sco, while Lesh's $M_{v}$ is ar average of eight stars, including $\omega^{1}$ Sco. The latter value is adopted.

The resulting new luminosity calibration for spectral types 03-B5, luminosity class III - V, is listed in table 1 and illustrated in Fig. 2. It is recommended that this calibration be used for modern spectral classification on the MK system.

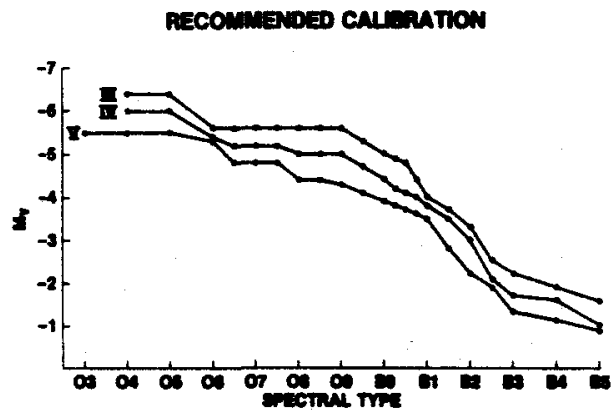

Fig. 2. Recommended calibration 
TABLE I

RECOMMENDED CALIBRATION

\begin{tabular}{|c|c|c|c|}
\hline $\begin{array}{l}\text { Spectral } \\
\text { Type }\end{array}$ & \multicolumn{3}{|c|}{ Luminosity Class } \\
\hline $\begin{array}{l}03 \\
04 \\
05 \\
06 \\
06.5 \\
07 \\
07.5 \\
08 \\
08.5 \\
09 \\
09.5 \\
\text { B0 } \\
\text { B0.2 } \\
\text { B0.5 } \\
\text { B0.7 } \\
\text { B1 } \\
\text { B1.5 } \\
\text { B2 } \\
\text { B2 .5 } \\
\text { B3 } \\
\text { B4 } \\
\text { B5 }\end{array}$ & $\begin{array}{c}V \\
-5.5 \\
-5.5 \\
-5.5 \\
-5.3 \\
-4.8 \\
-4.8 \\
-4.8 \\
-4.4 \\
-4.4 \\
-4.3 \\
-4.1 \\
-3.9 \\
-3.8 \\
-3.7 \\
-3.6 \\
-3.5 \\
-2.8 \\
-2.2 \\
-1.9 \\
-1.3 \\
-1.1 \\
-0.9\end{array}$ & $\begin{array}{l}\text { IV } \\
-6.0 \\
-6.0 \\
-5.4 \\
-5.2 \\
-5.2 \\
-5.2 \\
-5.0 \\
-5.0 \\
-5.0 \\
-4.7 \\
-4.4 \\
-4.2 \\
-4.1 \\
-4.0 \\
-3.8 \\
-3.5 \\
-3.0 \\
-2.1 \\
-1.7 \\
-1.6 \\
-1.0\end{array}$ & $\begin{array}{l}\text { III } \\
-6.4 \\
-6.4 \\
-5.6 \\
-5.6 \\
-5.6 \\
-5.6 \\
-5.6 \\
-5.6 \\
-5.6 \\
-5.3 \\
-5.0 \\
-4.9 \\
-4.8 \\
-4.4 \\
-4.0 \\
-3.7 \\
-3.3 \\
-2.5 \\
-2.2 \\
-1.9 \\
-1.6\end{array}$ \\
\hline
\end{tabular}


REFERENCES

Blaauw, A. (1963). In Basic Astronomical Data, K. Aa. Strand, ed., University of Chicago Press: Chicago, Chap. XX.

Conti, P.S. and Alschuler, W.R. (1971). Astrophys. J. 170, 325.

Hiltner, W.A., Garrison, R.F. and Schild, R.E. (1969). Astrophys. Lesh, J.R. $(1968)$. Astrophys. J. Suppl. 17, 371.

Snow, T.P. and Morton, D.C. (1976). Astrophys. J. Suppl. 32, 429. Walborn, N.R. (1971). Astrophys. J. Suppl. 23, 257.

Walborn, N.R. (1972). Astron. J. 77, 312.

Walborn, N.R. (1973). Astron. J. 73, 1067. 


\section{DISCUSSION}

Garrison: $\delta$ Sco is above the main sequence at BO.5. What distance modulus did you use for Upper Scorpius?

Lesh: The spectral type of $\delta$ Sco is B0.5IV. Because of the "diagonal effect" in spectral classification, it should have about the same absolute magnitude as $\tau$ Sco, which is BOV. The fact that it lies so far above $T$ Sco in Snow and Morton's diagram is due to an inconsistency in the calibration.

As for the distance modulus for Scorpius, my 1968 calibration was based on the photometry of Borgman and Blaauw, which ultimately goes back to Bertiau's individual astrometric distances for Scorpio-Centaurus stars. However, at BOV, where the absolute magnitude must be set by either $\tau$ Sco or $U$ Ori, I have chosen the latter, because I consider the distance modulus for Orion to be better determined than that for Scorpio-Centaurus. 